\title{
Effets De L'incorporation Du Son De Maïs A Différents Doses Sur Les Performances De Croissance Des Lapereaux Et La Rentabilité De L'élevage
}

\author{
Tchibozo Sélidji Vital Chlodulfe (Master en Agronomie) \\ Dossa Luc Hippolyte (PhD, Maître de Conférences) \\ Houndonougbo Fédéric (PhD, Maître de Conférences) \\ Koura Bossima Ivan (PhD, Assistant) \\ Houinato Benjamin Marcel (PhD, Professeur Titulaire)
}

Ecole des Sciences et Techniques de Production Animale, Faculté des

Sciences Agronomiques, Université d'Abomey-Calavi, Cotonou, Benin.

doi: 10.19044/esj.2017.v13n24p473 URL:http://dx.doi.org/10.19044/esj.2017.v13n24p473

\begin{abstract}
This study was conducted to evaluate the performance of rabbits fed with rations containing corn bran at different levels of incorporation. Three rations, R5, R11 and R16, were formulated with incorporation rates of corn bran of $5.5 \%, 11 \%$ and $16.5 \%$, respectively. Ninety young rabbits of 5 weeks of age were used for the experiments. The fattening study lasted 8 weeks and that of digestibility lasted for 1 week. The findings showed that the ration with $5.5 \%$ maize bran showed the best growth performances (18 $\mathrm{g} / \mathrm{d})$ with the highest digestibility values of dry matter $(67 \%)$ and cellulose (97\%). The ration with $16.5 \%$ corn bran yields $17 \mathrm{~g} / \mathrm{d}$ with a better digestibility of the crude protein $(97 \%)$ and the highest efficiency index (2.40). The ration with $11 \%$ maize bran is the most expensive (1395 $\mathrm{Fcfa} / \mathrm{kg}$ ) and has intermediate characteristics to that of the other two. The ration $\mathrm{R}$ could be used by rabbit farmers in urban and peri-urban areas to improve animal zootechnical performances. Future studies should be focused on the digestive use of different nutrients in rabbits through an in vivo study.
\end{abstract}

Keywords: Rabbit, Ration, Performance, Maize bran

\section{Resume}

Une étude a été conduite pour évaluer les performances des lapins nourris avec des rations comportant du son de maïs à différentes taux d'incorporation. Trois rations R5, R11 et R16 ont été formulées avec des 
taux d'incorporation de son de maïs de 5,5\%, $11 \%$ et $16,5 \%$, respectivement. Quatre-vingt-dix lapereaux de 05 semaines d'âge ont été utilisés pour les expérimentations. L'étude d'engraissement a durée 08 semaines et celle de digestibilité, 01 semaine. Les résultats montrent que la ration avec $5,5 \%$ de son de maïs présente les meilleures performances de croissance (18 g/jr) avec les valeurs les plus élevées de digestibilité de la matière sèche $(67 \%)$ et de cellulose $(97 \%)$. La ration avec $16,5 \%$ de son de maïs permet des gains de poids de $17 \mathrm{~g} / \mathrm{jr}$ avec une meilleure digestibilité de la protéine brute $(97 \%)$ et l'indice d'efficience alimentaire la plus élevée $(2,40)$. La ration avec $11 \%$ de son de maïs est la plus cher $(1395 \mathrm{Fcfa} / \mathrm{kg})$ et présente des caractéristiques intermédiaires à celle des deux autres. La ration R5 peut être utilisée par les éleveurs de lapins en zone urbaine et périurbaine, pour de bonnes performances zootechniques. Des études futures devraient s'intéresser à l'utilisation digestive des différents nutriments chez le lapin, à travers une étude in vivo.

Mots-clés: Lapins, Ration, Performance, Son de maïs

\section{Introduction}

La production carnée au Bénin est en nette croissance (FAO, 2015) et la cuniculture semble être un moyen très simple de fournir de la protéine animale à moindre coût à la population tant urbaine que rurale. Néanmoins, une difficulté majeure dans ce secteur est le coût élevé des aliments. Le coût alimentaire dépend directement de facteurs tels que la disponibilité des matières premières, le coût de ces matières premières, etc. Le maïs, matière première utilisée en alimentation animale est la céréale la plus consommée tant en alimentation humaine qu'animale en Afrique subsaharienne. Au niveau mondial, les deux tiers du maïs produit sont utilisés pour l'alimentation animale, $27 \%$ pour 1'alimentation humaine. On observe ainsi une compétition entre les hommes et les animaux pour le maïs en Afrique subsaharienne et principalement au Bénin. Pour remédier à ce problème il est nécessaire de trouver une alternative à l'utilisation du maïs en alimentation animale. Il s'agira de trouver une formulation permettant de maintenir ou même d'augmenter les performances des animaux tout en réduisant le coût alimentaire et le taux d'incorporation du maïs en intégrant le son de maïs, les autres facteurs de productions étant supposés fixes. C'est dans cette dynamique que s'inscrit ce travail qui vient suggérer la substitution du maïs par le son maïs dans l'alimentation des lapins pour parvenir à l'optimisation des coûts alimentaire. 


\section{Materiel Et Methodes}

\section{Cadre d'étude}

L'expérimentation a été conduite dans la commune de Tori-Bossito, situé entre $6^{\circ} 30^{\prime} 11^{\prime}$ 'de latitude Nord et $2^{\circ} 08^{\prime} 42^{\prime}$ 'de longitude Est. Le climat y est de type subéquatorial avec deux saisons pluvieuses alternées de deux saisons sèches de durée inégales. Les hauteurs moyennes de pluies enregistrées par la Direction National de la Météorologie entre 1984 et 2013 sont entre $900 \mathrm{~mm}$ et $1450 \mathrm{~mm}$. Le sol est de type ferralitique (Volkoff, 1976).

\section{Son de Maïs}

Le son de maïs conditionné par la société Maïserie du Bénin (SOUSIA) a été utilisé pour l'expérimentation. Il comporte $88,70 \%$ de matière sèche, $43,80 \%$ d'amidon et $10,10 \%$ de protéines brutes.

\section{Animaux}

Quatre-vingt-dix (90) lapereaux de 05 semaines d'âge, avec un poids moyen de $834 \pm 08 \mathrm{~g}$ ont été utilisés pour l'expérimentation. Avant l'essai, ils ont été traités contre les parasites internes et externes et contre la coccidiose. Aussi, de la vitamine leur a été offert.

\section{Dispositif Expérimental}

L'expérimentation s'est déroulée de Juin à Août 2015 dans une ferme cunicole dans la commune de Tori. Le dispositif expérimental est un bloc aléatoire complet avec 03 traitements (rations), 05 répétitions et 06 lapereaux par répétition.

Les lapins ont été élevés dans des clapiers où les normes d'élevage (aération, ventilation, abreuvoirs, mangeoires, etc.) ont été largement respectées. La densité d'élevage qui a été appliquée pour la mise en lots est de 10 sujets $/ \mathrm{m}^{2}$.

L'aliment expérimental est constitué d'une ration conventionnelle de lapin avec le son de maïs à différent taux d'incorporation. Trois rations R5, R11 et R16 ont été formulées avec des taux d'incorporation de son de maïs de $5,5 \%$; $11 \%$ et $16,5 \%$, respectivement. Les proportions des différents ingrédients dans les rations formulées sont présentées dans le Tableau 1.

Tableau 3. Ingrédients inclus dans les trois rations formulées

\begin{tabular}{cccc}
\hline Matières & $\mathbf{R 5}$ & $\mathbf{R 1 1}$ & $\mathbf{R 1 6}$ \\
Premières (\%) & & & \\
\hline Maïs & 16.5 & 11 & 5.5 \\
Son de maïs & $\mathbf{5 . 5}$ & $\mathbf{1 1}$ & $\mathbf{1 6 . 5}$ \\
Son de blé & 28.58 & 28.58 & 28.58 \\
Tourteau de soja & 9 & 9 & 9 \\
\hline
\end{tabular}




\begin{tabular}{cccc}
\hline Tourteau de coton & 7 & 7 & 7 \\
Tourteau de palmiste & 30 & 30 & 30 \\
Coquille & 1.7 & 1.7 & 1.7 \\
Lysine & 0.1 & 0.1 & 0.1 \\
Méthionine & 0.1 & 0.1 & 0.1 \\
Phosphate bicalcique & 1 & 1 & 1 \\
NaCl & 0.3 & 0.3 & 0.3 \\
Premix & 0.2 & 0.2 & 0.2 \\
Sulfate de fer & 0.02 & 0.02 & 0.02 \\
\hline
\end{tabular}

$\mathrm{R} 5$ : ration avec 5,5\% de son de maïs, R11 : ration avec $11 \%$ de son de maïs, R16= ration avec $16,5 \%$ de son de maïs.

L'aliment a été distribué sous forme granulés aux lapereaux 2 fois/jour (à 8 heures et à 16 heures) dans des mangeoires avec les bords repliés pour réduire les pertes par gaspillage et de l'eau a été offert à volonté dans des abreuvoirs.

\section{Collecte des Données}

Après une pesée individuelle des animaux au début de l'expérimentation, les animaux sont pesés hebdomadairement durant les six (08) semaines d'engraissement. Les quantités d'aliments servies et le refus du jour précédent ont été pesés chaque jour.

\section{Paramètres Calculés}

Le gain moyen quotidien (GMQ) a été calculé comme le changement de poids vif entre deux mesures successives divisés par le nombre de jours entre les deux mesures. Le taux de conversion alimentaire (TCA) a été estimé comme le gain (ou perte) en poids (en g) divisé par la quantité d'aliment consommée (en g). l'Indice d'efficience alimentaire a été estimé comme le gain généré par le GMQ divisé par le coût de l'aliment consommé (Houndonougbo et al., 2012).

La digestibilité apparente a été calculé comme la différence entre la quantité d'aliment ingérée et celle dans les fèces comme une proportion de l'aliment ingéré, exprimé en pourcentage.

\section{Traitement des Données}

Les données ont été analysées selon la procédure GLM du logiciel statistique SPSS Inc. (2010). La comparaison multiple des moyennes a été effectuée ainsi qu'un test de significativité pour déterminer les différences en les rations servies aux animaux. 


\section{Resultats}

\section{Composition chimique de la ration}

Le Tableau 2 présente la composition chimique des rations testées. L'analyse de ce tableau révèle que la R16 a les plus grandes valeurs de protéine brute et de cellulose comparées aux autres rations. Toutefois cette ration présente les plus faibles teneurs en amidon, en $\mathrm{Ca}$ et $\mathrm{P}$.

Tableau 2. Composition chimique des différentes rations testées

\begin{tabular}{cccc}
\hline Paramètres & $\mathbf{R}_{\mathbf{5}}$ & $\mathbf{R}_{\mathbf{1 1}}$ & $\mathbf{R}_{\mathbf{1 6}}$ \\
\hline Matières sèches & 78,9 & 82,1 & 87,08 \\
Matières grasses & 8,7 & 9,8 & 8,04 \\
Amidon & 13,08 & 18,05 & 12,1 \\
Cellulose brute & 14,02 & 13,9 & 15,09 \\
Protéine brute & 16,02 & 14,6 & 16,20 \\
$\mathrm{Ca}$ & 8,07 & 12,5 & 10,03 \\
$\mathrm{P}$ & 6,08 & 5,7 & 5,1 \\
$\mathrm{Na}$ & 2,9 & 2,6 & 2,2
\end{tabular}

$\mathrm{R} 5$ : ration avec 5,5\% de son de maïs, R11 : ration avec $11 \%$ de son de maïs, $\mathrm{R} 16=$ ration avec $16,5 \%$ de son de maïs.

\section{Performances des animaux et Rentabilité économique}

Le Tableau 3 présente les performances pondérales, l'ingestion alimentaire et les performances économiques des lapins pour les différentes rations testées.

Tableau 4. Effet du son de maïs à différentes doses sur l'ingestion alimentaire, le gain de poids et l'indice d'efficience alimentaire des rations

\begin{tabular}{c|rrrrr}
\hline \multicolumn{1}{c}{ Paramètres } & \multicolumn{1}{c}{ R5 } & R11 & R16 & $\begin{array}{r}\text { Erreur } \\
\text { standard }\end{array}$ & Pr>F \\
\hline Poids initial (g) & $1370,16^{\mathbf{A}}$ & $1301,48^{\mathbf{B}}$ & $1291,05^{\mathbf{B}}$ & 15,06 & 0,001 \\
Poids final (g) & $1496,07^{\mathbf{A}}$ & $1414,42^{\mathbf{B}}$ & $1409,57^{\mathbf{B}}$ & 16,33 & 0,001 \\
Gain Moyen Quotidien (g/jr) & 17,99 & 16,13 & 16,93 & 1,680 & NS \\
& & & & & \\
Consommation Alimentaire (g/jr de & $72,92^{\mathbf{A}}$ & $72,88^{\mathbf{A}}$ & $69,58^{\mathbf{B}}$ & 0,600 & 0,001 \\
1'aliment) & & & & & \\
Ingestion de MS & $57,53^{\mathbf{B}}$ & $59,84^{\mathbf{A}}$ & $60,59^{\mathbf{A}}$ & 0,340 & 0,001 \\
Ingestion d'amidon & $9,54^{\mathbf{B}}$ & $13,16^{\mathbf{A}}$ & $8,42^{\mathbf{C}}$ & 0,060 & 0,001 \\
Ingestion de cellulose & $10,22^{\mathbf{B}}$ & $10,13^{\mathbf{B}}$ & $10,50^{\mathbf{A}}$ & 0,060 & 0,001 \\
Ingestion de PB & $11,68^{\mathbf{A}}$ & $10 ., 641^{\mathbf{C}}$ & $11 ., 27^{\mathbf{B}}$ & $0 ., 067$ & $0 ., 001$ \\
Taux Convertion Alimentaire & 0,26 & 0,23 & 0,26 & 0,020 & $\mathrm{NS}$ \\
Coût Alimentaire (FCFA aliment/ & 1090,26 & 1394,21 & 1118,90 & 228,61 & $\mathrm{NS}$ \\
poids vif) & & & & & \\
Indice Efficience Alimentaire & 2,30 & 2,12 & 2,40 & 0,21 & $\mathrm{NS}$ \\
\hline
\end{tabular}


$\mathrm{MS}=$ matière sèche, $\mathrm{PB}=$ Protéine brute, $\mathrm{R} 5:$ ration avec $5,5 \%$ de son de maïs, $\mathrm{R} 11$ : ration avec $11 \%$ de son de maïs, R16= ration avec $16,5 \%$ de son de maïs. Erreur standard : différence significative entre moyennes sur la même ligne (A, B, C: $p<0.01)$.

Pour la plupart des paramètres calculés, aucune différence n'a été observée entre les moyennes pour les trois rations. Le gain moyen quotidien est en moyenne de 17,02 $\pm 1,68 \mathrm{~g} / \mathrm{jr}$, le taux de conversion alimentaire de $0,25 \pm 0,023$, le coût alimentaire de $1201 \pm 229$ FCFA aliment/poids vif et l'indice d'efficience alimentaire de 2,27 $\pm 0,21$.

Il existe une différence significative entre la consommation alimentaire et l'ingestion alimentaire pour les trois rations. Elle est statistiquement $(\mathrm{p}<0,001)$ plus élevée au niveau des rations $\mathrm{R} 0$ et $\mathrm{R} 1$ comparée à la ration R2 $(70 \mathrm{~g} / \mathrm{jr})$. L'ingestion de l'amidon est élevée ( $\mathrm{p}<$ $0,001)$ en R11 (13 g/jr) et faible en R16 ( $8 \mathrm{~g} / \mathrm{jr})$. Celle de la protéine brute est élevée ( $\mathrm{p}<0,001)$ en R5 (12 g/jr) et faible en R 11 ( $11 \mathrm{~g} / \mathrm{jr})$. L'ingestion de la cellulose est en moyenne de $10 \mathrm{~g} / \mathrm{jr}$, quoiqu'elle est statistiquement plus élevée $(p<0,001)$ en R16.

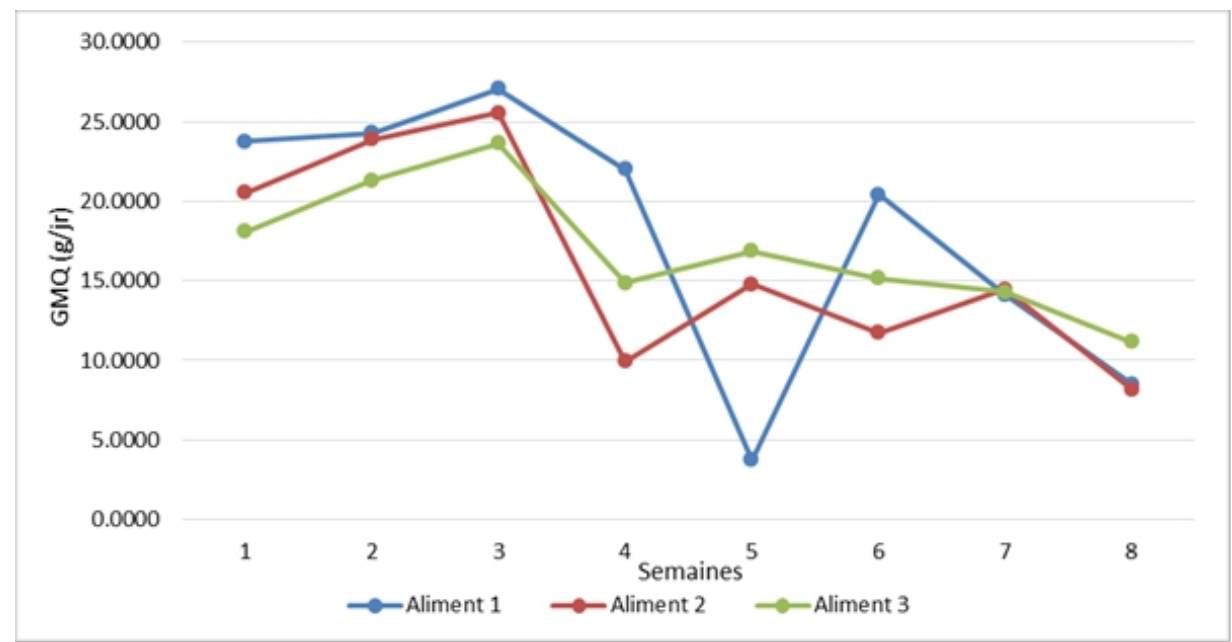

Figure 4. Evolution du GMQ au cours de la période d'engraissement

La Figure 1 montre l'évolution du GMQ des animaux au cours de la période d'engraissement. De la $1^{\text {ère }}$ à la $3^{\text {e }}$ semaine, on note une augmentation du GMQ de tous les animaux quel que soit la ration. Néanmoins, on remarque nettement que durant cette période, les animaux soumis à la ration R0 ont les meilleures performances suivis de ceux soumis à la ration $\mathrm{R} 1$ (GMQ $>25 \mathrm{~g})$. A partir de la $3^{\mathrm{e}}$ semaine, une baisse des GMQ $(-5$ à $-20 \mathrm{~g} / \mathrm{jr})$ s'observe. De la $4^{\mathrm{e}}$ à la $5^{\mathrm{e}}$ semaine la tendance décroissance s'observe toujours au niveau de la ration 1 qui atteint sa valeur la plus faible, alors qu'une reprise de poids s'observe pour les rations R1 et R2. De la $5^{\mathrm{e}}$ à la $6^{\mathrm{e}}$ semaine, le GMQ relatif à la ration $\mathrm{R} 0$ croît à nouveau au-dessus 
des autres rations. Au cours des deux dernières semaines, les GMQ au niveau de la ration 0 décroissent et reste proche de ceux des rations $\mathrm{R} 1$ et $\mathrm{R} 2$, avec des valeurs de $20 \mathrm{~g} / \mathrm{jr}$.

\section{Digestibilité de l'aliment}

Le Tableau 4 présente la digestibilité des aliments. Une différente significative est observée entre les digestibilités de $\mathrm{N}$ et de la cellulose d'une ration à l'autre. La digestibilité de $\mathrm{N}$ est plus élevée $(\mathrm{p}<0.01)$ pour la ration $\mathrm{R} 16$ (97) comparée aux autres rations, 93\% et 93\% pour R5 et R11, respectivement. Au contraire, la ration R16 avait la digestibilité de la cellulose la plus faible ( $\mathrm{p}<0.01$ ), $92 \%$ contre $97 \%$ et $97 \%$ respectivement pour R5 et R11.

Tableau 4. Effet du son de maïs à différentes doses sur l'ingestion $(\mathrm{g} / \mathrm{jr})$ et la digestibilité $(\%)$ de la matière sèche, de la protéine et la cellulose

\begin{tabular}{crrrrr}
\hline Paramètres & R5 & R11 & R16 & $\begin{array}{r}\text { Erreur } \\
\text { standard }\end{array}$ & Pr>F \\
\hline Ingestion MS (g) & 60,03 & 56,08 & 51,16 & 8,74 & NS \\
dMS & 67,23 & 60,18 & 60,46 & 8,36 & NS \\
Ingestion PB (g) & 12,19 & 9,97 & 9,52 & 1,61 & NS \\
dPB & $93,34^{\mathbf{B}}$ & $93,46^{\mathbf{B}}$ & $97,14^{\mathbf{A}}$ & 1,072 & 0,010 \\
Ingestion Cellulose (g) & 10,67 & 9,49 & 8,87 & 1,50 & $\mathrm{NS}$ \\
dCellulose & $97,06^{\mathbf{A}}$ & $97,00^{\mathbf{A}}$ & $91,94^{\mathbf{B}}$ & 1,29 & 0.010 \\
\hline
\end{tabular}

$\mathrm{MS}=$ matière sèche, $\mathrm{PB}=$ Protéine brute, $\mathrm{R} 5$ : ration avec $5,5 \%$ de son de maïs, R11 : ration avec $11 \%$ de son de maïs, R16= ration avec $16,5 \%$ de son de maïs. Erreur standard : différence significative entre moyennes sur la même ligne (A, B, C: p < 0.01).

\section{Discussion}

L'alimentation des lapins est l'objet d'attention scientifique depuis des décennies en Afrique au sud du Sahara. En effet, les élevages de ces herbivores rongeurs, source de protéine animale et de revenus, disparaissent parce que peu rentables du fait des coûts alimentaires de plus en plus élevés. Dans l'optique de réduire les coûts alimentaires, le recours à des ressources non conventionnelles localement disponibles, tels que les sons de céréales a été souvent mentionnés (Luisa Falc̃ao-e-Cunha et al., 2004; Oduguwa et al., 2008). Toutefois les performances des lapins avec des rations comportant forte valeur de son de maïs ont été peu investiguées.

En général, notre étude montre que les gains de poids des animaux sont en accord avec la composition chimique des rations et les ingestions. Les résultats de digestibilité sont très variables. Les GMQ sont relativement plus élevés $(18 \mathrm{~g} / \mathrm{jr})$ avec seulement $5 \%$ de taux d'incorporation du son de maïs dans la ration des lapins. Des GMQ de $17 \mathrm{~g} / \mathrm{jr}$ sont attendus avec la ration comportant $16,5 \%$ de son de maïs.

Ces GMQ sont relativement plus faibles comparés à ceux obtenus dans la littérature (19,7 à 23,1 g/jr) par (Koura et al., 2015) en incorporant à 
$10 \%$ des enveloppes de gousse de niébé $(22 \mathrm{~g} / \mathrm{jr}$ ), par (Houndonougbo et al., 2012) en utilisant les tourteaux de palme et (Kpodekon et al., 2009) en utilisant une ration conventionnelle de lapin.

La ration R16 présente une forte ingestion de la matière sèche et des nutriments. Le taux de conversion alimentaire était aussi élevé avec cette ration, quoique la différence ne soit pas significative avec les autres rations. Ceci suggère tout de même une meilleure valorisation de cet aliment par le lapin. Toutefois les valeurs de IEA obtenu sont faibles comparées aux résultats obtenus dans la littérature par (Houndonougbo et al., 2012; Kpodekon et al., 2009; Adeyemi et al., 2010; et Koura et al., 2015). Aussi, les coûts alimentaires sont relativement plus élevés que ceux obtenus par (Koura et al., 2015) en incorporant les résidus de récolte.

La digestibilité des rations suit la tendance des ingestions. La protéine est la mieux digérée avec la ration R16 alors que la cellulose en est la moins digeste. Toutefois les résultats de digestibilité de la matière sèche obtenus sont relativement faibles comparés à ceux de (Aboh et al., 2013) avec les épluchures d'ananas; (Etchu et al., 2013) avec les fanes d'arachide et (Koura et al., 2015) avec les enveloppes de gousses de niébé. Les résultats de digestibilité de la protéine et de la cellulose sont relativement très élevés (91-97\%) dans notre étude. Ceci peut être dû aux différences dans la teneur en fibre des différents ingrédients substitués. Selon (Gidenne \& Bellier, 2000), la digestibilité de la matière sèche augmente avec la teneur en fibre.

\section{Conclusion}

Notre étude sur l'engraissement des lapins avec des rations comportant du son de maïs à différentes taux d'incorporation révèle que la ration avec $5,5 \%$ de son de maïs présente les meilleures performances de croissance (18 g/jr) avec les valeurs les plus élevées de digestibilité de la matière sèche et de cellulose. La ration avec $16,5 \%$ de son de maïs permet des gains de poids de $17 \mathrm{~g} / \mathrm{jr}$ avec une meilleure digestibilité de la protéine brute et l'indice d'efficience alimentaire la plus élevée. La ration avec $11 \%$ de son de maïs est la plus cher et présente des caractéristiques intermédiaires à celle des deux autres. Les rations avec de faible teneur en son de maïs paraissent plus adaptées pour les petits producteurs dans nos villes et campagnes.

\section{Remerciements}

Les auteurs remercient le PROCAD pour avoir financé ce travail à travers le Projet PPAAO (Programme de productivité agricole en Afrique de l' Ouest). 


\section{References:}

1. Aboh A.B., Olaafa M., Dossou-Gbété G.S.O., Dossa A.D., and Djagoun N. (2002). Ingestion volontaire et digestibilité apparente d'une ration à base de la farine de graines de Mucuna pruriens var. utilis complétée de fourrages chez les lapins. Tropicultura, 20: 165169

2. Adedeji O.S., Amao S.R., Ameen S.A., Adedeji T.A. and Ayandiran T.A. (2013). Effects of Varying Levels of Leucaena leucocephala Leaf Meal Diet on the Growth Performance of Weaner Rabbit. Journal of Environmental Issues and Agriculture in Developing Countries, 5: 5-9.

3. Akoegninou A., van der Burg W.J., van der Maesen L.J.G., Adjakidje V., Essou J.P., Sinsin B. and Yedomonhan H. (2006). Analytical Flora of Benin (1st edn). Backhuys Publishers: Cotonou (Benin) \& Wageningen.

4. Dahouda M., Adjolohoun S., Senou M., Toleba S.S., Abou M., Vidjannagni D.S., Kpodekon M. and Youssao A.K.I. (2013). Effets des aliments contenant les folioles de Moringa oleifera Lam. et des aliments commerciaux sur les performances de croissance des lapins (Oryctolagus cuniculus) et la qualité de la viande. International Journal of Biological and Chemical Sciences, 7(5): 1838-1852.

5. Etchu K. A., Ngu G.T., Yongabi K.A., and Woogeng I.N. (2013). Effect of varying level of groundnut (Arachis Hypogea) haulms on the growth Performance of weaners rabbits (Oryctalagus cuniculus). International journal of livestock production, (5):81-87.

6. Falc ${ }^{\sim}$ ao-e-Cunha L., Peres H., Freire J.P.B., and Castro-Solla L. (2004). Effects of alfalfa, wheat bran or beet pulp, with or without sunflower oil, on caecal fermentation and on digestibility in the rabbit. Animal Feed Science and Technology, 117: 131-149.

7. Gidenne T. and Bellier R. (2000). Use of digestible fibre in replacement to available carbohydrates - Effect on digestion, rate of passage and caecal fermentation pattern during the growth of the rabbit. Livestock Production Science, 63: 141-152.

8. Houndonougbo M.F., Chrysostome C.A.A.M., Attakpa S.E., Sezan A. and Dehou H.B. (2012). Growth Performance of Rabbits Fed Palm-Press Fibres-Based Diets. International Scholarly Research Network (ISRN), Veterinary Science, DOI:10.5402/2012/915729.

9. Koura B.I., Houndonougbo F. and Houinato M. (2015). Effect of incorporation of cowpea and soybean pods in diets on feed intake, Digestibility and weight gain performances of rabbit. Revue CAMES, Science de la vie, de la terre et agronomie, 03(02): 32-36. 
10. Kpodekon M., Youssao A.K.I., Koutinhouin G.K., Baba L., Dessou J.M. and Djago Y. (2009). Effet de la granulation sur les performances de croissance, l'efficacité alimentaire et la viabilité des lapereaux en condition d'élevage tropical. Revue d'Élevage et Médecine Vétérinaire des Pays Tropicaux, 62 (1): 75-80.

11. Oduguwa O.O., Edema M.O., Ayeni A.O. (2008). Physico-chemical and microbiological analyses of fermented corn cob, rice bran and cowpea husk for use in composite rabbit feed. Bioresource Technology, 99: 1816-1820.

12. Volkoff B. (1976). Carte pédologique de reconnaissance à 1:200 000 de la République Populaire du Bénin. ORSTOM. Paris. 